\title{
When to Cryopreserve Ovarian Tissue: Effects of Chemotherapy on the Ovarian Reserve by Studying Follicular Density, Tissue Damage and Follicular Apoptosis
}

\section{Elsa Labrune ( $\nabla$ elsa.labrune@chu-lyon.fr)}

Université Claude Bernard Lyon 1: Universite Claude Bernard Lyon 1

\section{Serge Bianchetti}

Universite Claude-Bernard: Universite Claude Bernard Lyon 1

\section{Odile Lepinasse}

Civil Hospices of Lyon: Hospices Civils de Lyon

\section{Gaelle Soignon}

Civil Hospices of Lyon: Hospices Civils de Lyon

\section{Bruno Salle}

Universite Claude Bernard Lyon 1 Faculte de medecine Lyon-Sud: Universite Lyon 1 Faculte de Medecine et de Maieutique Lyon-Sud Charles Merieux

\section{Jacqueline Lornage}

Universite Claude Bernard Lyon 1 Faculte de medecine Lyon-Sud: Universite Lyon 1 Faculte de Medecine et de Maieutique Lyon-Sud Charles Merieux

\section{Research}

Keywords: Fertility preservation, Chemotherapies, Ovarian tissue, Follicular density, Apoptosis

Posted Date: September 20th, 2021

DOl: https://doi.org/10.21203/rs.3.rs-885722/v1

License: (c) (i) This work is licensed under a Creative Commons Attribution 4.0 International License. Read Full License 


\section{Abstract}

\section{Background}

Patients scheduled to receive chemotherapy should be counseled on fertility preservation. Known gonadotoxic chemotherapies such as alkylating agents have a high risk of altering ovarian reserve. In some cases, the urgency of treatment requires the use of chemotherapy before fertility preservation, which will be carried out at a later stage. Most often the ovarian tissue is cryopreserved. The aim of our study is to investigate the impact of chemotherapies on follicular density, tissue damage and apoptosis of reserve follicles.

\section{Results}

We included 140 patients: 63 patients, mean age 18.8 years, were included in the group no chemotherapy (group A) and 77 patients, mean age 17.1 years, in the group presence of chemotherapy before ovarian conservation (group B). None of the patients had had pelvic radiotherapy prior to ovarian cryopreservation. The histological parameters studied were: follicular density, the presence of cortical fibrosis and the presence of vessel abnormalities. We selected 12 patients from group $A$ and 15 patients from group $B$, comparable in age and pathology, for whom we evaluated follicle apoptosis by immunostaining cleaved caspase 3 . We demonstrated an inverse relationship between follicular density and age $(p<0.0001)$, as well as a lack of effect of chemotherapy on follicular density $(p=0.87)$. There was no difference in other histological parameters. On the other hand, we showed an impact of chemotherapies, especially alkylating agents, on the apoptosis of ovarian follicles $(p<0.0001)$.

\section{Conclusion}

Our study is the largest cohort reported to date. This work underlines that conservation of ovarian tissue after chemotherapy remains possible.

\section{Background}

Patients scheduled to receive chemotherapy should be counseled on fertility preservation. Known gonadotoxic chemotherapies such as alkylating agents have a high risk of altering ovarian reserve (1). In some cases, the urgency of treatment requires the use of chemotherapy before fertility preservation, which will be carried out at a later stage. Most often the ovarian tissue is cryopreserved. There are several fertility preservation strategies for women undergoing potentially gonadotoxic treatment: embryo cryopreservation, oocyte cryopreservation and ovarian tissue cryopreservation. To date, more than 200 live births have been published after a procedure of slow freezing of ovarian tissue fragments, thawing and then orthotopic grafting (2-5). This procedure is the reference method in terms of ovarian tissue preservation. Two live births have been reported after self-grafting of ovarian tissue cryopreserved by vitrification (6). Since the technique of freezing fragments of human ovarian tissue in ART laboratories has been used in current practice, numerous studies have attempted to compare the effect of 
chemotherapy prior to ovarian sampling on follicular density (7-11) and to search for tumor cells in these fragments, whose presence is a contraindication to transplantation (12-19). The first publications studied the effect of complete chemotherapy (i.e. composed of induction, consolidation, and maintenance) on follicular reserve: Himelstein-Braw et al. found a deleterious effect of chemotherapy on macroscopic examination of the ovary after autopsy of children who died of leukemia (20). In addition, Marcello et al. studied the structure of patients in remission after chemotherapy treatment for acute lymphoblastic leukemia using optical and electron microscopy. Light microscopy showed in all cases cortical fibrosis, sometimes so severe that no follicles were observed. For the majority of patients, the total number of follicles seemed to be reduced. The ultrastructural study revealed abnormalities of the stroma, particularly of the capillaries, and an absence of follicular abnormality (21). Finally, Familiari et al. analyzed the ovarian tissue of patients treated for Hodgkin's disease by electron microscopy: abnormalities were found with vacuolation of oocytes and granulosa cells, associated with thickening of the basement membrane (22).

When the procedure for freezing fragments of ovarian tissue appeared, several publications were published on the population of patients who had received chemotherapy prior to ovarian cryopreservation. The aim of these studies was to highlight, or not, one or more differences (by optical and/or electron microscopy) between this population and the population of patients who did not receive chemotherapy prior to cryopreservation (control group). For patients receiving chemotherapy prior to ovarian retrieval, the chemotherapy protocols vary greatly in intensity (from a single dose of induction chemotherapy to full chemotherapy, with the patient being referred by the onco-hematologists when the disease progresses or recurs); some authors have found no histological difference between the chemotherapy group and the control group, free of chemotherapy, prior to ovarian cryopreservation $(7,8$, 11). On the contrary, other publications have found histological differences between these two groups (11). Meirow et al. also studied ovarian tissue from patients operated for ovarian cryopreservation before potentially sterilizing chemotherapy. In the group of patients who started chemotherapy, several abnormalities were present: blood vessel wall abnormalities, anarchic neovascularization in the ovarian cortex, focal cortical fibrosis. According to the authors, all of these abnormalities are responsible for focal ischemia, which contributes to the decrease in ovarian reserve. However, in the control group, the elderly patients presented these same anomalies (9).

The main objective of our study was the histological study of ovarian fragments from 140 patients referred to the department of reproductive medecine of the Lyon hospitals between August 1997 and March 2014 for cryopreservation. We compared the histological criteria of follicular density, the presence of cortical fibrosis and the appearance of vessels in women who had not received chemotherapy prior to cryopreservation versus those who had received treatments. The secondary objective of the study was to compare follicle apoptosis within the ovarian cortex fragment in 12 treatment-naïve women versus 15 women who received one or more courses of chemotherapy.

\section{Results}




\subsection{Description of the population}

Of the 140 patients included in the study, 63 patients had not received treatment prior to ovarian cryopreservation (group A) and 77 patients had received treatment prior to ovarian self-conservation (group B). In Group A, 40 patients were referred prior to cancer treatment, 15 patients were referred for treatment of non-malignant hematological diseases, 7 patients were referred for treatment of autoimmune diseases, and 1 patient had a genetic disease.

The 40 cancers consisted of 14 Hodgkin's diseases, 6 Ewing's sarcomas, 4 sarcomas, 1 neuroblastoma, 2 non-Hodgkin's malignant lymphomas, 1 breast cancer, 3 adenocarcinomas, 2 myelodysplasias and 2 mesotheliomas.

Non-malignant hemopathies were represented by 6 sickle cell diseases, 2 thalassemias, 3 idiopathic bone marrow aplasias, 3 Fanconi diseases, and 1 agranulocytosis. The 7 autoimmune diseases were composed of 2 lupus, 1 vasculitis, 1 idiopathic thrombocytopenic purpura, 1 sarcoidosis, 1 scleroderma and 1 severe combined immune deficiency. Finally, the genetic disease was a Turner syndrome, with a mosaicism of $8 \%$, which had had spontaneous puberty associated with normal growth but showed a progressive decrease in $\mathrm{AMH}$.

Group B, consisting of 77 patients, was composed of 75 cancers and 2 non-malignant hematological diseases. The 75 cancers were 17 Hodgkin's diseases, 4 Ewing's sarcomas, 17 acute leukemias, 11 neuroblastomas, 9 non-Hodgkin's malignant lymphomas, 4 renal cancers, 4 medulloblastomas, 2 chronic myeloid leukemias, and 7 other cancers.

Finally, the 2 non-malignant hemopathies were major thalassemia and idiopathic bone marrow aplasia.

Group A is composed of more non-malignant hematopathies and autoimmune diseases than group B. On the other hand, group B had more malignant hematological diseases, including acute leukemia and neuroblastoma: pathologies that require emergency chemotherapy. As for the treatments received by the patients in group $B$, none of them included pelvic radiotherapy. 55 patients had been treated with chemotherapy containing alkylating agents. Of these 55 patients, 9 patients had been treated with the ABVD protocol.

\subsection{Follicular density study}

The mean follicular density was 8.8 follicles $/ \mathrm{mm}^{2}$ (ranges: $0-266.7$ ). The number of ovarian biopsies without follicles on histological examination was comparable in both groups $(9.7 \%$ in group A versus $12.7 \%$ in group $B, p=0.501$ ). Follicular density was significantly inversely related to patient age $(p<0.0001)$. The higher the age of the patient, the more the follicular density decreased (Figure 1). This result was observed in all groups.

The mean \pm standard deviation of the follicular density of group A was 9.1 follicles $/ \mathrm{mm}^{2} \pm 33.8$ (ranges: 0-266) and 9.9 follicles $/ \mathrm{mm}^{2} \pm 18.6$ (ranges: $0-75$ ) for group B (Figure 2). It is important to note that the 
average age of the two groups was comparable $(18.8 \pm 9.5$ years for group A and $17.1 \pm 9.4$ years for group B). There was therefore no statistically significant difference in follicular density between the two groups ( $p=0.863)$. Chemotherapy treatments did not alter follicular density. The subgroup of patients who received alkylating agents consisted of 55 patients of whom 9 had received ABVD. The mean age of this subgroup was $17.0 \pm 9.6$ years. The mean \pm standard deviation follicular density of the patients who received alkylating agents, which was 8.8 follicles $/ \mathrm{mm}^{2} \pm 14.8$, was comparable to that of group $A$ $(p=0.960)$. Seven patients had no follicles during histological analysis with a higher mean \pm standard deviation age of 20 years \pm 9.7 .

The mean follicular density of group A was not statistically different from that of group B across age subgroups $(p=0.638)$. There was no effect of chemotherapy; there was only an age effect $(p=0.001)$, age being a known factor impacting follicular density (Figure 3 ).

\subsection{Study of other histological parameters}

Regarding the appearance of the vessels and the presence of cortical fibrosis of the ovarian tissue, the number of biopsies with these abnormalities was identical in both groups. In Group A, 8 biopsies had vessel abnormalities, which were statistically comparable to Group $B$, where 5 biopsies had the same abnormalities (12.7\% vs. 6.5\%). Similarly, cortical fibrosis was described in 4 patients in Group A versus 5 patients in Group B (6.3\% vs. $6.5 \%)$.

Three patients had malignant medullar infiltration: in 2 of them (1.5\% of patients), the infiltration was found to be malignant (lymphocyte infiltration in non-Hodgkin's malignant lymphoma, and lymphoblast infiltration in acute chronic myeloid leukemia). Only one infiltration was benign (lymphoplasmocyte infiltration without significance in lupus).

Of the 2 patients with histology finding malignant cells, one was 25 years old at the time of cryopreservation and had acute chronic myeloid leukemia in acute lymphoblastic leukemia. She had been treated with AISA (other inhibitors of DNA synthesis) prior to ovarian cryopreservation. The second patient was 20 years old at the time of cryopreservation, had diffuse CD20+ large B-cell lymphoma with osteomedullary localization. The patient did not receive chemotherapy prior to ovarian cryopreservation.

\subsection{Study of ovarian follicle apoptosis by caspase 3 cleavage staining}

In order to study the impact of chemotherapies on ovarian follicle apoptosis, we performed cleaved caspase 3 staining in 12 patients in group $A$ and 15 patients in group B. We matched patients on age, which is an important factor in follicular reserve, and on the type of pathology. The patient groups were comparable (Table 1). The mean age in the "no chemotherapy" group was $19.5 \pm 8.7$ years (ranges: $3-31$ ) and the mean age in the "presence of chemotherapy" group was $19.9 \pm 7.5$ years (ranges: 6-31) $(p=0.904)$. The "no chemotherapy" group consisted of 7 hematological malignancies and 4 solid tumors. The "presence of chemotherapy" group consisted of 9 hematological malignancies and 5 solid tumors. Each group was composed of one benign hematological pathology. 
We analyzed 202 follicles from patients in the "no chemotherapy" group and 291 ovarian follicles in the "presence of chemotherapy" group. The mean \pm standard deviation number of follicles observed per patient was 16.8 follicles \pm 9.2 in the "no chemotherapy" group and $18.2 \pm 11.5$ in the "presence of chemotherapy" group $(p=0.741)$. The percentage of positive follicles in the "no chemotherapy" group was $5.9 \%$ vs. $15.1 \%$ in the "presence of chemotherapy" group ( $p<0.01)$. Apoptosis was significantly greater in the group receiving chemotherapy prior to ovarian cryopreservation.

In the "presence of chemotherapy" group, 161 follicles were derived from 6 patients who received nonABVD alkylating agents before ovarian cryopreservation. The alkylating agents were cyclophosphamide, ifosfamide and/or oxaliplatin. The percentage of positive follicles was $22.9 \%$ in this group versus $5.9 \%$ in the no chemotherapy group $(p<0.0001)$. The presence of alkylating agents, outside the ABVD protocol, resulted in a significant increase in apoptosis in ovarian follicles.

83 follicles from the group that received ABVD prior to ovarian cryopreservation were analyzed. 2 follicles were positive, representing $2.4 \%$ of the follicles. The use of ABVD did not result in more apoptosis than that observed in the group without chemotherapy.

Finally, the other chemotherapies did not seem to increase the apoptosis of the ovarian follicles. In fact, 5 follicles were positive out of the 47 follicles read, i.e. $10.6 \%$ of the follicles, or twice the percentage of follicular apoptosis of group A. The lack of significance may be related to the low number of follicles analyzed.

It is interesting to note that follicular apoptosis was always present in the granulosa cells. We did not observe any positive oocyte alone.

\section{Discussion}

The aim of our study was to evaluate the reserve follicles of ovarian tissues of patients before or after treatment with chemotherapies. To do this, we first studied the follicular density of the patients as well as the following histological parameters: appearance of the vessels and presence of cortical fibrosis. Then we evaluated the role of treatments on the apoptosis of ovarian follicles. Our study evaluating the impact of chemotherapy on ovarian non-growing follicle is the largest cohort reported to date. In fact, we describe a group of 77 patients who received chemotherapy prior to ovarian cryopreservation among the 140 patients included. It is also a study that describes the apoptosis of ovarian follicles according to treatments in the context of female fertility preservation.

We have demonstrated a link between the age of the patients and the follicular density. Herein, as age increases, the follicular density decreases. This correlation has been described in several articles $(7,11,12)$. On the other hand, the Seshadri team in 2006 did not find a link between follicular density and age. In their study, the total number of patients was low $(n=26)$ with a high mean age (22 years old) and a range that was not representative of the different age groups, as the youngest patient was 13 and the oldest 29 (8). 
We have also shown the absence of effect of chemotherapies, especially alkylating agents, on follicular density and on the histological aspect of ovarian tissue. These results corroborate those found by different authors $(7,8,10,11)$. On the contrary, Oktem and Oktay described a statistically significant decrease in ovarian reserve in the presence of chemotherapies containing alkylating agents. However, the numbers were low $(n=5)$ and the mean age of the group receiving alkylating agents was high (29.8 years old) (12). Meirow et al. described an impact of chemotherapy on ovarian tissue. They reported neovascularization and fibrosis in patients previously treated with chemotherapy. Neovascularization was demonstrated by immunohistochemical markers (anti-CD31 and anti-CD34), which we did not perform in our study (9). The absence of effect of the treatments on follicular density does not mean the absence of qualitative alterations in the ovarian follicles: follicular alterations were observed as intracytoplasmic oocyte vacuole, nuclear abnormalities of granulosa cells (10). These anomalies can be explained by an entry in apoptosis of the follicles. We evaluated the apoptosis of follicles according to the presence or absence of chemotherapy and found a significant increase in apoptosis of the follicles of women previously treated with chemotherapy and more particularly chemotherapies containing alkylating agents. These results corroborate those found by Soleimani et al. and Li et al $(23,24)$. Soleimani et al. and then $\mathrm{Li}$ et al. studied the effect of doxorubicin and cyclophosphamide in ovarian xenografts $(23,24)$. Recently a team has demonstrated another phenomenon of follicular loss; they showed follicular activation of reserve follicles after chemotherapy with alkylating agents. The number of growing follicles was increased a few days after treatment (25). We did not observe this phenomenon, which is probably due to the time between treatment and preservation of ovarian tissue, which was longer in our cohort by several months. They did not observe an increase in follicular apoptosis immediately after chemotherapy. Another important point is the age of the patients, they were all pubescent (25) unlike our study where the average age is lower. It would be interesting to evaluate this hypothesis on ovarian tissue from non-pubertal girls.

We have demonstrated a lack of effect of chemotherapies on this follicular density associated with an increase in follicular apoptosis. Chemotherapies did not quantitatively alter the ovarian follicles but probably qualitatively. Our results were in line with the data of Luan's team; she showed that cyclophosphamide, which is an alkylating agent, did not decrease the number of primordial follicles but activated the signaling pathways of apoptosis (26). The effect on these follicles could be modified after the transplant. It would be interesting to compare post-graft apoptosis with that previously performed on fresh tissue to evaluate the repair mechanisms that may have been established. It is indirectly demonstrated by the live birth rate after ovarian tissue transplantation in patients who have received chemotherapy. This live birth rate was not affected by the history of chemotherapy.(5).

To complete this work on the study of apoptosis, it would be interesting to increase the total number of patients in the study and therefore the number of patients per chemotherapy category, and the number of pre-pubertal patients. The time between the last chemotherapy course and the surgical removal of ovarian tissue should also be studied in order to evaluate the time required for chemotherapies to induce apoptosis in the ovarian follicles. The study of apoptosis by caspase 3 cleaved labelling is a technique that allows the evaluation of the triggering or not of apoptotic phenomena by chemotherapies in a global 
way. Its more refined analysis could be envisaged using a DNA chip. The apoptotic pathway in question could be targeted, as well as receptors, intracellular modulators and transcription factors. It would also be interesting to study the quality of the vessels, as gonadotoxic treatments may alter these vessels which are essential for the recovery of ovarian cortex grafts after surgery. Here too, the study of endothelial cell apoptosis would be appropriate.

In view of these results it would be logical to carry out cryopreservation of ovarian tissue before any treatment when possible. This work underlines the fact that patients treated with chemotherapy before ovarian cryopreservation can benefit from this fertility preservation technique. However, the use of alkylating agents prior to preservation surgery should be avoided. This should be discussed according to the clinical context because a balance must be found between the risk of invasion of ovarian tissue by malignant cells without the use of chemotherapies before ovarian conservation, and the use of alkylating agents that can avoid this invasion but alter the ovarian tissue. It is essential to remind oncology teams of the importance of referring patients promptly to reproductive medicine departments in order to preserve patients' fertility in the most optimal conditions.

\section{Methods}

\subsection{Population}

Between August 1997 and March 2014, 220 patients benefited from ovarian cryopreservation in the context of fertility preservation in the department of reproductive medicine of the Lyon hospitals. Patients whose anatomopathological analysis was not performed in our center were excluded. Likewise, patients whose data was incomplete were excluded. We therefore included 140 patients in our study. We defined 2 groups: a group A composed of patients who had not received any treatment prior to cryopreservation and a group $B$ composed of patients who had received one or more potentially gonadotoxic treatments prior to ovarian cryopreservation. Group A, consisting of 63 patients with a mean age of $18.8 \pm 9.5$ years (1-34 years), was comparable to group $B$, which contained 77 patients with a mean age of $17.1 \pm 9.41$ years (1-35 years). We retrieved the treatment protocols for the patients in group $B$. The chemotherapy molecules received were classified into 5 categories: alkylating agent (alkylating), topoisomerase inhibitor (TI), antimetabolites (AM), other inhibitors of DNA synthesis (AISA), and immunosuppressants (IS). None of the patients had had pelvic radiotherapy prior to ovarian cryopreservation.

\subsection{Histologic study}

During the dissection of the ovary, according to our laboratory freezing procedure (27), a fragment of cortex and a fragment of medullary are systematically sent to the anatomy and pathological laboratory in order to count the ovarian follicles over the whole cortex, to study the appearance of the tissue and to look for tumor cells invasion. After fixation in formaldehyde 4\% (VWR, Strasbourg, France) at room temperature, the fragments are included in paraffin, then cut into $4 \mu \mathrm{m}$ serial sections with a microtome. Six slices were performed, spaced of $60 \mu \mathrm{m}$, and stained with Hematoxylin (Millipore, Burlington, USA), Eosin (Sigma-Aldrich, St Quentin Fallavier, France), Safran (RAL diagnostics, Martillac, France) (HES) 
stain. The parameters studied were: the follicular density of the reserve follicles that are not growing, the presence of cortical fibrosis and the presence of vessel abnormalities (wall thickening). Vessel abnormalities were a thickening of the vessel wall with a fibrohyalinosis appearance. We collected all these data from the pathology reports except the follicular density which was calculated by relating the total number of follicles present on the histological slide to the total area of the biopsy. All follicles regardless of stage were counted in double blind by a pathologist and an embryologist (Figure 4). The results obtained are expressed as the number of follicles per $\mathrm{mm}^{2}$. When on the anatomopathological report there was mentioned tumor cells, we considered the immunohistochemical results.

\subsection{Study of apoptosis}

Among the 140 patients included, we selected 12 patients from group $A$ and 15 patients from group $B$, comparable in age and pathology. Among the 15 patients in group B, 6 patients had received alkylating agents outside the ABVD protocol (the alkylating agents were cyclophosphamide and ifosfamide), 5 patients had been treated by the ABVD protocol and finally 4 patients had received chemotherapy containing no alkylating agent. We separated the patients treated with ABVD chemotherapy from the group of patients treated with alkylating agents because the dose of alkylating agent in the ABVD protocol is lower. This ABVD treatment is described by oncologists as low gonadotoxic (28). For all these patients, we performed two slices, spaced of $60 \mu \mathrm{m}$, that we stained with the caspase 3 cleaved kit (Asp $175, \neq 9661$, Cell Signaling Technology, Danvers, USA) according to their protocol. This marking allowed us to evaluate the apoptosis of the follicles within the tissue. We read the slides in double-blind under a confocal fluorescence microscope. On average, 18.3 follicles were read per slide (ranges: 2-43). We performed a positive control which was a histological thymus slide (tissue with very high apoptosis) (Figure 5) and a negative control which was a slide of ovarian tissue on which we did not deposit the primary antibody (Figure 5). A follicle was considered positive when the whole follicle showed a fluorescence of more than 50\% homogeneously (Figure 5) or when the oocyte is fluorescent.

\subsection{Statistical analysis}

The statistical analyses were performed using the software " $\mathrm{R}$ " version 2.15.2. The comparison of two percentages was carried out by a Chi 2 test. The comparison of two averages was performed by a Student $t$-test and the comparison of more than two averages was performed by an ANOVA test. An analysis of variance was performed to study the follicular density according to the age of the patients. Test results are considered significant if the $p$ is less than 0.05 .

\section{Abbreviations}

AISA: other inhibitors of DNA synthesis

TI: topoisomerase inhibitor

AM: antimetabolites 
IS: immunosuppressants

ABVD: doxorubicine-bleomycine-vinblastine-dacarbazine

DNA: desoxyribonucleic acid

HES: hematoxylin-eosin-safran

\section{Declarations}

Ethics approval and consent to participate: The study was validated by the local ethics committee (Hospices Civils de Lyon). All patients included in the study signed a written consent after receiving informed information. All the procedures adhered to relevant ethical regulations.

Consent for publication: Not applicable.

Availability of data and materials: The datasets used and/or analysed during the current study are available from the corresponding author on reasonable request.

Competing interests: The authors declare that they have no competing interests.

Funding: This research did not receive any specific grant from funding agencies in the public, commercial, or not-for-profit sectors.

Authors' contributions: Participation in design (EL, SB, BS, JL), data analysis (EL, SB, JL), execution and manuscript drafting (EL, SB, OL, GS) and critical discussion (EL, SB, OL, GS, BS, JL).

Acknowledgements: Not applicable.

\section{References}

1. Oktay K, Harvey BE, Loren AW. Fertility Preservation in Patients With Cancer: ASCO Clinical Practice Guideline Update Summary. J Oncol Pract. 2018;14(6):381-5.

2. Donnez J, Dolmans M-M. Fertility Preservation in Women. N Engl J Med. 2018;25(4):400-1. 378(.

3. Dolmans M-M, Falcone T, Patrizio P. Importance of patient selection to analyze in vitro fertilization outcome with transplanted cryopreserved ovarian tissue. Fertil Steril août. 2020;114(2):279-80.

4. Marin L, Bedoschi G, Kawahara T, Oktay KH. History, Evolution and Current State of Ovarian Tissue Auto-Transplantation with Cryopreserved Tissue: a Successful Translational Research Journey from 1999 to 2020. Reprod Sci avr. 2020;27(4):955-62.

5. Shapira M, Dolmans M-M, Silber S, Meirow D. Evaluation of ovarian tissue transplantation: results from three clinical centers. Fertil Steril août. 2020;114(2):388-97.

6. Suzuki N, Yoshioka N, Takae S, Sugishita Y, Tamura M, Hashimoto S, et al. Successful fertility preservation following ovarian tissue vitrification in patients with primary ovarian insufficiency. Hum 
Reprod mars. 2015;30(3):608-15.

7. Poirot C, Vacher-Lavenu M-C, Helardot P, Guibert J, Brugières L, Jouannet P. Human ovarian tissue cryopreservation: indications and feasibility. Hum Reprod juin. 2002;17(6):1447-52.

8. Seshadri T, Gook D, Lade S, Spencer A, Grigg A, Tiedemann K, et al. Lack of evidence of disease contamination in ovarian tissue harvested for cryopreservation from patients with Hodgkin lymphoma and analysis of factors predictive of oocyte yield. Br J Cancer 10 avr. 2006;94(7):100710.

9. Meirow D, Dor J, Kaufman B, Shrim A, Rabinovici J, Schiff E, et al. Cortical fibrosis and blood-vessels damage in human ovaries exposed to chemotherapy. Potential mechanisms of ovarian injury. Hum Reprod juin. 2007;22(6):1626-33.

10. Abir R, Ben-Haroush A, Felz C, Okon E, Raanani H, Orvieto R, et al. Selection of patients before and after anticancer treatment for ovarian cryopreservation. Hum Reprod avr. 2008;23(4):869-77.

11. Fabbri R, Vicenti R, Macciocca M, Pasquinelli G, Lima M, Parazza I, et al. Cryopreservation of ovarian tissue in pediatric patients. Obstet Gynecol Int. 2012;2012:910698.

12. Oktem O, Oktay K. Quantitative assessment of the impact of chemotherapy on ovarian follicle reserve and stromal function. Cancer 15 nov. 2007;110(10):2222-9.

13. Abir R, Feinmesser M, Yaniv I, Fisch B, Cohen IJ, Ben-Haroush A, et al. Occasional involvement of the ovary in Ewing sarcoma. Hum Reprod juill. 2010;25(7):1708-12.

14. Dolmans M-M, Marinescu C, Saussoy P, Van Langendonckt A, Amorim C, Donnez J. Reimplantation of cryopreserved ovarian tissue from patients with acute lymphoblastic leukemia is potentially unsafe. Blood 21 oct. 2010;116(16):2908-14.

15. Dolmans M-M, Jadoul P, Gilliaux S, Amorim CA, Luyckx V, Squifflet J, et al. A review of 15 years of ovarian tissue bank activities. J Assist Reprod Genet mars. 2013;30(3):305-14.

16. Dolmans M-M, Luyckx V, Donnez J, Andersen CY, Greve T. Risk of transferring malignant cells with transplanted frozen-thawed ovarian tissue. Fertil Steril mai. 2013;99(6):1514-22.

17. Rosendahl M, Andersen MT, Ralfkiær E, Kjeldsen L, Andersen MK, Andersen CY. Evidence of residual disease in cryopreserved ovarian cortex from female patients with leukemia. Fertil Steril nov. 2010;94(6):2186-90.

18. Bittinger SE, Nazaretian SP, Gook DA, Parmar C, Harrup RA, Stern CJ. Detection of Hodgkin lymphoma within ovarian tissue. Fertil Steril févr. 2011;95(2):803.e3-6.

19. Bastings L, Beerendonk CCM, Westphal JR, Massuger LF, a. G, Kaal SEJ, van Leeuwen FE, et al. Autotransplantation of cryopreserved ovarian tissue in cancer survivors and the risk of reintroducing malignancy: a systematic review. Hum Reprod Update oct. 2013;19(5):483-506.

20. Himelstein-Braw R, Peters H, Faber M. Morphological study of the ovaries of leukaemic children. Br J Cancer juill. 1978;38(1):82-7.

21. Marcello MF, Nuciforo G, Romeo R, Di Dino G, Russo I, Russo A, et al. Structural and ultrastructural study of the ovary in childhood leukemia after successful treatment. Cancer 15 nov. 
1990;66(10):2099-104.

22. Familiari G, Caggiati A, Nottola SA, Ermini M, Di Benedetto MR, Motta PM. Ultrastructure of human ovarian primordial follicles after combination chemotherapy for Hodgkin's disease. Hum Reprod déc. 1993;8(12):2080-7.

23. Soleimani R, Heytens E, Darzynkiewicz Z, Oktay K. Mechanisms of chemotherapy-induced human ovarian aging: double strand DNA breaks and microvascular compromise. Aging (Albany NY) août. 2011;3(8):782-93.

24. Li F, Turan V, Lierman S, Cuvelier C, De Sutter P, Oktay K. Sphingosine-1-phosphate prevents chemotherapy-induced human primordial follicle death. Hum Reprod janv. 2014;29(1):107-13.

25. Shai D, Aviel-Ronen S, Spector I, Raanani H, Shapira M, Gat I, et al. Ovaries of patients recently treated with alkylating agent chemotherapy indicate the presence of acute follicle activation, elucidating its role among other proposed mechanisms of follicle loss. Fertility and Sterility [Internet]. 20 janv 2021 [cité 28 avr 2021];0(0). Disponible sur: https://www.fertstert.org/article/S0015-0282(20)32715$1 /$ abstract.

26. Luan Y, Edmonds ME, Woodruff TK, Kim S-Y. Inhibitors of apoptosis protect the ovarian reserve from cyclophosphamide. J Endocrinol 1 févr. 2019;240(2):243-56.

27. Demirci B, Lornage J, Salle B, Frappart L, Franck M, Guerin JF. Follicular viability and morphology of sheep ovaries after exposure to cryoprotectant and cryopreservation with different freezing protocols. Fertil Steril avr. 2001;75(4):754-62.

28. Lee SJ, Schover LR, Partridge AH, Patrizio P, Wallace WH, Hagerty K, et al. American Society of Clinical Oncology recommendations on fertility preservation in cancer patients. J Clin Oncol. 20 juin 2006;24(18):2917-31.

\section{Tables}

Table 1: Comparison of the population in the two groups 
Group A (without chemotherapy)
Group B

(chemotherapy)

15

$19.9 \pm 7.5(p=0.904)$

6-31
Range (years)

Hematological malignancies ( $\mathrm{n}$ )

Hodgkin lymphoma

B cell lymphoma

Myelodysplasia

Acute leukemia

Solid tumors ( $\mathrm{n}$ )

Ewing sarcoma

Neuroblastoma

Rectal adenocarcinome

Nephroblastoma

Benign hematological pathologies (n)

Idiopathic thrombocytopenic purpura

Idiopathic medullar aplasia
12

$19.5 \pm 8.7$

3-31

7

5

1

1

0

4

2

1

1

0

1

1

0

0
9

6

1

0

2

5

2

1

1

1

1

1

\section{Figures}




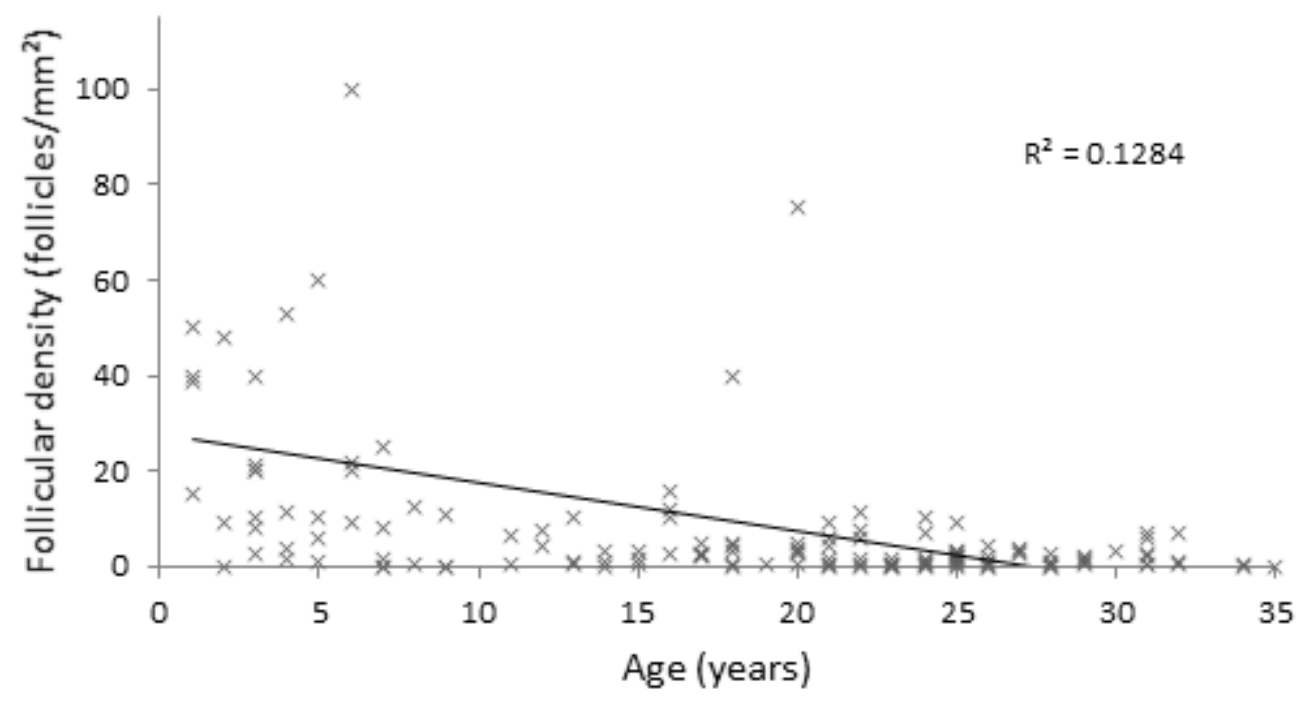

\section{Figure 1}

Distribution of follicular density (follicles $/ \mathrm{mm}^{2}$ ) according to the age of the patients (years)

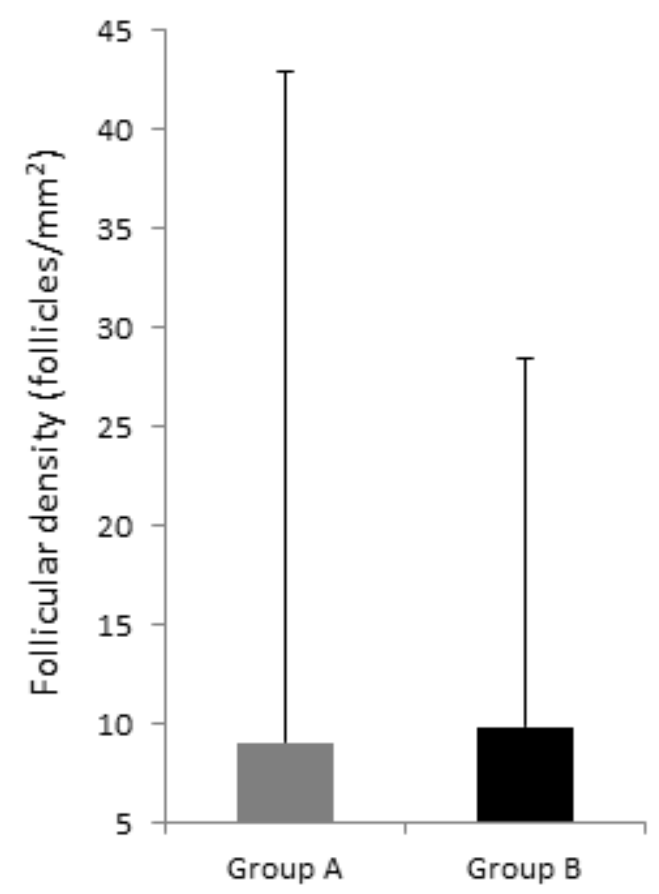

\section{Figure 2}

Follicular density (follicles $/ \mathrm{mm}^{2}$ ) according to groups: group A (without chemotherapy), group B (with chemotherapy before cryopreservation of ovarian cortex) 


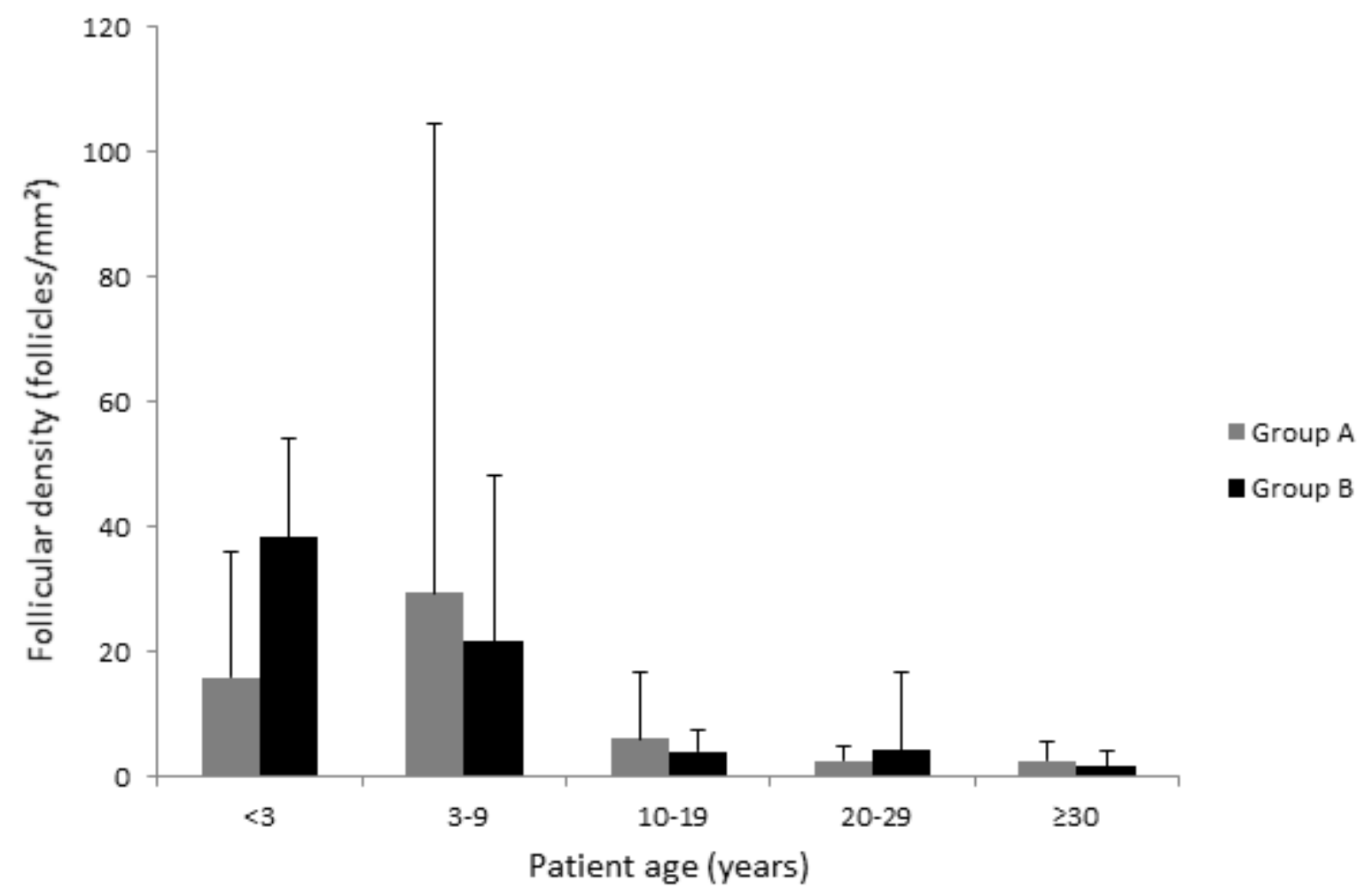

Figure 3

Follicular density (follicles $/ \mathrm{mm}^{2}$ ) by treatment group (group A: without chemotherapy, group B: chemotherapy before cryopreservation of ovarian cortex) in different age subgroups

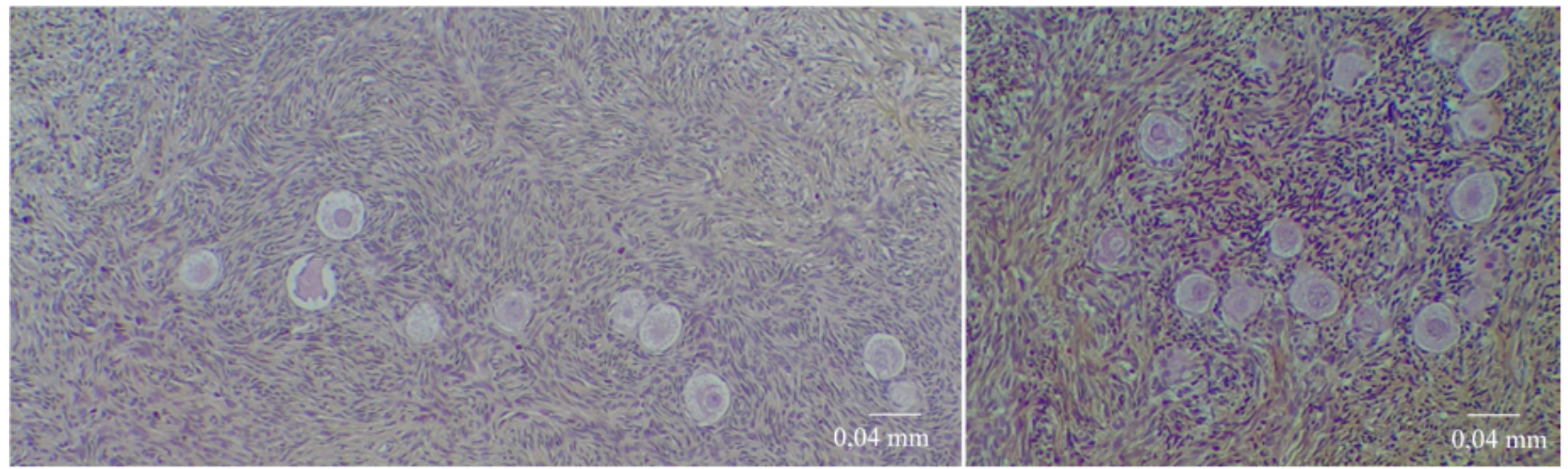

Figure 4

Section of human ovarian tissue stained with HES; primordial and primary follicles (white arrow); A: no chemotherapy before preservation; B: chemotherapy before preservation 


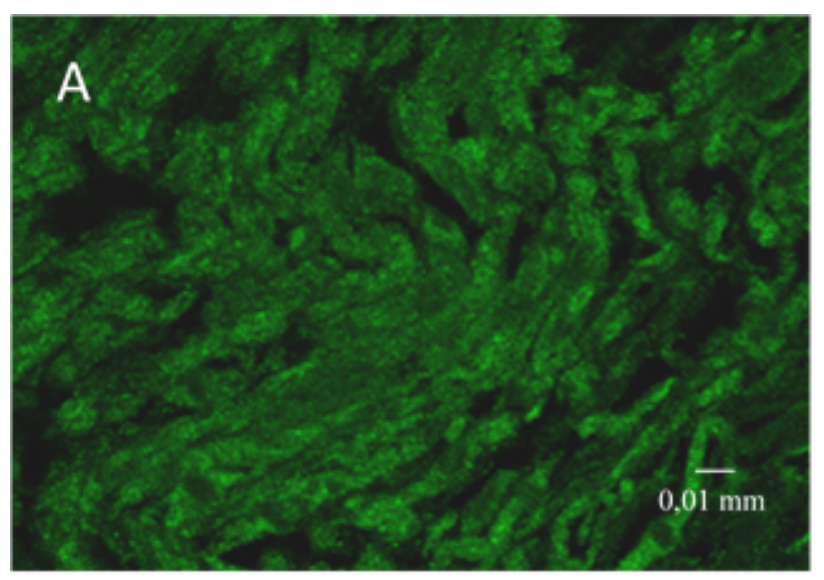

B
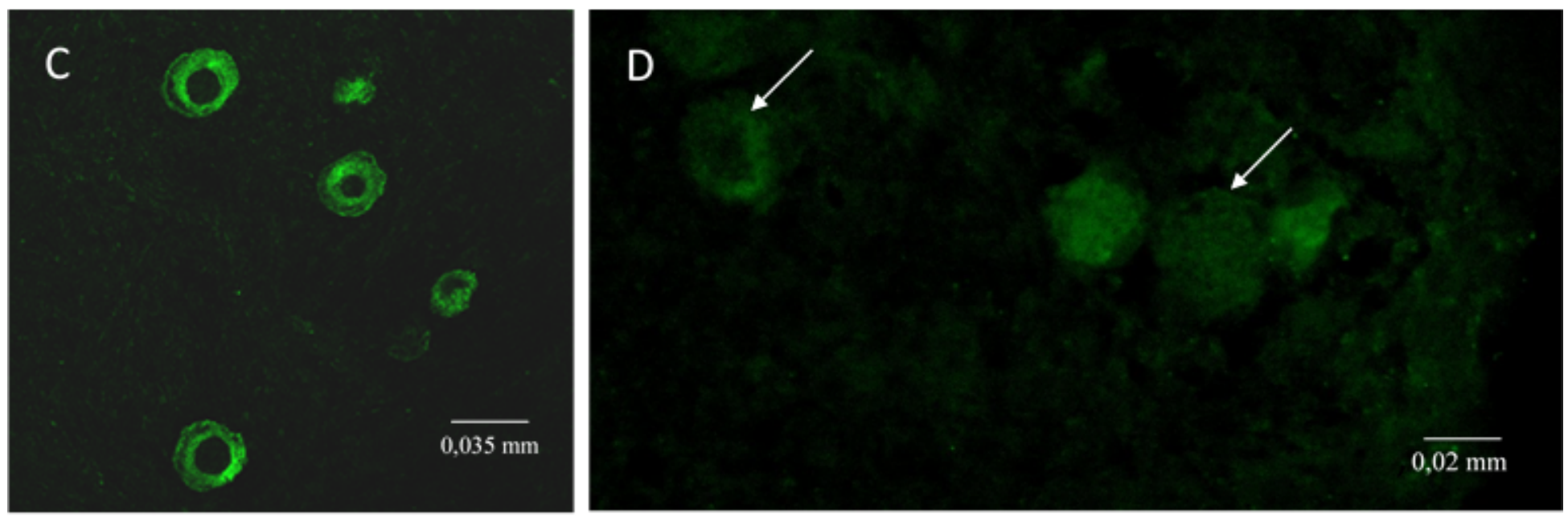

\section{Figure 5}

Ovarian follicle apoptosis by caspase 3 cleavage staining: A- Section of human thymus stained with an anti-cleaved caspase 3 antibody, positive control, magnification $400 \mathrm{~B}$-Section of human ovarian cortex without primary antibody, negative control, magnification $400 \mathrm{C}$ - Section of human ovarian cortex who received alkylating agents before preservation with ovarian follicles labeled with an anti-cleaved caspase 3 antibody, magnification 400 D- Section of human ovarian cortex who has not received chemotherapy prior to preservation with follicles no labeled (white arrow), magnification 400 